\title{
Direct Laryngoscopy under Topical Anaesthesia and Superior Laryngeal Nerve Block for Laryngeal and Hypopharyngeal Lesions in ENT Patients
}

\author{
Dr Arti $^{1}$, Deep Jyoti ${ }^{* 2}$, Neelam Kumar ${ }^{3}$, Kunzes Dolma ${ }^{4}$, Padam Singh Jamwal ${ }^{5}$ \\ ${ }^{1,2}$ Registrar, ENT, GMC Jammu, India, ${ }^{3}$ Resident, Pathology, GMC, Jammu, India, ${ }^{4}$ Post Graduate, ENT, GMC, Jammu, \\ India, ${ }^{5}$ Professor, ENT, GMC, Jammu, India
}

*Corresponding author- Deep Jyoti; deepjyotimanhas82@gmail.com

Received 07 October 2019;

Accepted 21 October 2019;

Published 01 November 2019

\begin{abstract}
Introduction: Direct laryngoscopy is a common procedure being done by otolaryngologists under general anaesthesia for various pathologies of the larynx and hypopharynx. Regional anaesthesia using topical spray and bilateral superior nerve block is an alternative for the direct laryngoscopy. Aim: To evaluate the effect of bilateral block of superior laryngeal nerve (SLN) and topical anesthesia in direct laryngoscopy for diagnostic purpose as well as for taking biopsies from the larynx and hypopharynx. Materials And Methods: This is a prospective study done on 20 patients in age group of 50 to 70 years with laryngeal and hypopharyngeal growth. All these patients underwent diagnostic direct laryngoscopy with biopsy under regional block which include bilateral superior laryngeal nerve block with $2 \%$ lignocaine and topical anaesthesia of oral cavity and transtracheal through cricothyroid membrane with $10 \%$ lignocaine spray and $4 \%$ lignocaine respectively. $\underline{\text { Result: }}$ Success rate in our study was $85 \%$ in supraglottic, transglottic, pyriform growth and only $15 \%$ in glottis and postcricoid growth based on assessment of hemodynamic stability and patients comfortable score before and after the procedure under regional block. $\underline{\text { Conclusion: }}$ Direct Laryngoscopy under topical anesthesia and superior laryngeal nerve block for biopsy is a good alternative to general anesthesia for the patients presenting in ENT with laryngeal and hypopharyngeal pathologies.
\end{abstract}

Keywords: Supraglottic, hypopharyngeal, superior laryngeal nerve, block, topical anaesthesia.

\section{Introduction}

Direct laryngoscopy is a diagnostic procedure used by otorhinolaryngologists for the diagnosis of various pathologies involving larynx, oropharynx and hypopharynx. This procedure can cause discomfort to the patient as it is done in awake condition $^{[1]}$. This procedure usually performed by the anasthesiologists also. This procedure is usually performed under general anesthesia with adequate muscle relaxation and immobile cords in order to avoid movement of vocal cords, coughing, bucking during laryngoscopy and therby preventing injuries/complications. An additional challenge in anesthetic management is the relative short operative time of this procedure which requires rapid awakening of the patient from general anaesthesia and rapid return of muscle power and laryngeal reflux $^{[2]}$. There have been some recent developments in regional anesthesia of the larynx, that have resulted in number of innovative and refined option to practitioners. Regional anaesthesia has been commonly used by anaesthesiologist to achieve awake intubation while it is less commonly used by otolaryngologists. Regional anaesthesia can be used in the patients with difficult airway and also in those high risk patients where general anaesthesia cannot be used. However, only limited type of surgeries can be performed under regional anesthesia. In addition, blocks are also subject to various complications or failure ${ }^{[3,4]}$. Regional anesthesia of the larynx include combination of the bilateral superior laryngeal nerve block with topical anesthesia of oral cavity and transtracheal through cricothyroid membrane. Direct laryngoscopy under regional anaesthesia helps to perform diagnostic/ theraputic laryngoscopy as an in office procedure. This study was designed to evaluate the effect of bilateral block of superior laryngeal nerve (SLN) and topical anesthesia in direct laryngoscopy for diagnostic purpose as well as for taking biopsies from the laryngeal and pharyngeal lesions in patients presenting to ENT OPD. Hemodynamic parameters (pulse and blood pressure) and comfort score of patient before and during the procedure was also assessed.

\section{Method}

This study was conducted in the Department Of ENT in GMC, Jammu, on 20 patients over a period of 6 months from 1st January 2019 to 1 st July 2019. An informed and written consent was 
obtained from the patients. A good rapport was developed with patients and the procedure was explained. History regarding systemic disorder such as hypertension, diabetes mellitus, ischemic heart disease and congestive cardiac failure was taken. Thorough examination of cardiovascular system, respiratory system and airway was done.

\section{Inclusion criteria}

Patients with Supraglottic, Glottis, Transglottic, Hypopharyngeal growth with or without tracheostomy.

\section{Exclusion criteria}

A) Patients with Subglottic growth.

B) Patient not giving consent for direct laryngoscopy under local anesthesia.

\section{Technique}

Patients were shifted to the operating table and they were made to lie in the supine position. Pulse and blood pressure were recorded. An intravenous infusion was secured in one forearm. With the patient lying supine the head was maximally extended and the hyoid bone identified by palpation. Since hyoid bone does not articulate with any other bone, it is readily movable, and this mobility serves as a useful identifying sign. After cleansing the neck with iodophore, a 21 gauge needle was introduced laterally at the greater cornu of the hyoid. After contacting the bone, the needle was then moved caudally until it slipped off the hyoid, just through the thyrohyoid membrane, and in to a closed space bounded by thyrohyoid membrane laterally and laryngeal mucosa medially. This space contains the ramifications of the internal branch of the superior laryngeal nerve. A careful aspiration is done to see that no air or blood is aspirated. This helps us to ensure that the needle does not lie in the lumen of vessel or that of the larynx. 1-2 $\mathrm{ml}$ of $2 \%$ lignocaine was injected. The block was then repeated on another side in the same manner. $2 \mathrm{ml}$ of $4 \%$ lignocaine was injected via cricothyroid membrane puncture at the end of maximal expiration. As the anaesthetic agent enters the trachea, patient coughs thereby spreading anaesthetic agent throughout the trachea and supraglottis. Tip of the epiglottis and oropharynx may retain some sensitivity. Patient was asked to open his/her mouth and the tongue was gently held protruding out. A spray of $10 \%$ lidocaine was directed towards posterior pharyngeal wall. This prevented gagging while introducing the laryngoscope. Pulse and blood pressure was again recorded during the procedure in each patient. Glycopyrrolate injection given intramuscularly takes care of the excessive secretions. Patients were kept fasting 2 hours before and after the procedure.

The hyoid bone may be difficult to locate in short, fat neck. In such cases the block technique can be modified by identifying the superior cornu of the thyroid cartilage and moving the needle cephlad and insert the needle directly through the thyrohyoid membrane. Feeling of resistance, offered by the membrane indicate when it has been punctured. However, definite identification of either the hyoid bone, or superior cornu of the thyroid cartilage during the block provide a reliable indication of depth of the needle $e^{[5]}$.

Patient comfortable score6 during direct laryngoscopy included the following parameters:

No reaction

Slight grimacing

Heavy grimacing

Verbal objection

Defencing movement of head and hands

\section{Statistical Analysis}

All the data was analysed using software SPSS 17.0. Characteristics of the patients was analysed using the descriptive analysis.

\section{Results}

This study was conducted on 20 patients presenting with growth larynx among which 19 were male and one was female. All these patients were in the age group between 54 to 70 years with mean age of $62 \pm 5.6$ years. After giving block to the patients, hemodynamic parameters i.e pulse and BP were noted before and during the procedure. Table 1,2,3 shows the hemodynamic parameters in case of supraglottic, glottis and pyriform sinus growths respectively. We had 2 patients with trans-glottis growth and one patient with post cricoid growth.

Table 1: Haemodynamics of the patients in Supraglottic growth $(n=10)$

\begin{tabular}{|l|l|l|}
\hline \multirow{2}{*}{ Parameters } & Mean \pm SD \\
\cline { 2 - 3 } & Before procedure & During procedure \\
\hline Pulse rate & $75.44+-10.44$ & $90.6+-10.25$ \\
\hline SBP & $118.6+-10.28$ & $133.5+-10.40$ \\
\hline DBP & $76+-11.66$ & $87.5+-7.96$ \\
\hline
\end{tabular}

Table 2: Haemodynamics in the Pyriform sinus growth $(n=4)$

\begin{tabular}{|l|l|l|}
\hline \multirow{2}{*}{ Parameters } & Mean+-SD & \multicolumn{2}{|c|}{} \\
\cline { 2 - 3 } & Before procedure & During procedure \\
\hline Pulse rate & $78+-10.54$ & $87.5+-11.08$ \\
\hline SBP & $115+-15.59$ & $128.5+-2.28$ \\
\hline DBP & $74+-11.575$ & $84+-11.83$ \\
\hline
\end{tabular}

Table 3: Haemodynamics in the Glottic growth $(n=3)$

\begin{tabular}{|l|l|l|}
\hline \multirow{2}{*}{ Parameters } & Mean+-SD & \multicolumn{2}{|c|}{} \\
\cline { 2 - 3 } & Before procedure & During procedure \\
\hline Pulse rate & $76+-7.48$ & $96.67+-4.11$ \\
\hline SBP & $115.33+-3.40$ & $148.67+-8.15$ \\
\hline DBP & $72+-1.63$ & $98.33+-4.11$ \\
\hline
\end{tabular}

Table 4 shows patient comfortable score during the procedure. Patients were having hemodynamic stability and good comfortable score in supraglottic, transglottic, pyriform sinus growth thus allowing surgeon to access the growth and to take biopsy.

Table 4: Patient comfortable score during the direct laryngoscopy

\begin{tabular}{|l|l|l|l|l|l|}
\hline Parameter & Supraglottic growth & Glottic growth & Transglottic growth & Pyriform growth & Post cricoids growth \\
\hline No reaction & - & - & - & - & - \\
\hline Slight grimacing & 4 & - & 1 & 3 & - \\
\hline Heavy grimacing & 4 & 1 & 1 & - & 1 \\
\hline Verbal objection & 2 & - & - & - & - \\
\hline $\begin{array}{l}\text { Defencing movement of } \\
\text { head and hand }\end{array}$ & - & 2 & - & - & - \\
\hline
\end{tabular}


Success rate in our study was $85 \%$ (17 out of 20 patients). In remaining $15 \%$, i; e in glottis and postcricoid growth, direct laryngoscopy was abandoned as patients showed defencing movements and hemodynamic instability. In case of supraglottic growth, patients showed slight grimacing and were comparitively comfortable till the completion of the procedure. Similarly, in transglottic and pyriform growths also, patients showed slight grimacing and were comfortable during procedure.

\section{Discussion}

In the present study of 20 patients, direct laryngoscopy done using bilateral superior laryngeal nerve block along with topical anesthesia mostly consisted of older patients unfit for general anesthesia. Older patients are usually unfit for general anaesthesia due to multiple systemic disorders ${ }^{[7]}$. This procedure provides adequate anaesthesia for about 45- 60 minutes during which a detailed examination of the larynx can be done and adequate biopsy can be taken from any lesion. This technique is well tolerated in multiple injections in vocal folds during type 1 thyroplasty, $\mathrm{CO} 2$ laser ablasion of respiratory papillomatosis and soft tissue biopsy as well ${ }^{[8]}$. a similar type of local anaesthesia has been described for diagnostic endoscopy of the airway to the carina and in indirect laryngeal surgeries. The superior laryngeal block was not used in such procedures ${ }^{[9,10]}$. These tools prove to be useful not only in operating room setting but also in emergency room and intensive care area as well and also add to the confidence and abilities of the practioner ${ }^{[11]}$. Direct laryngoscopy was successful in $85 \%$ of cases in our study which is nearer to the success rate reported by study done by Sivakumar et al ${ }^{[7]}$ with $82.5 \%$ of success and low failure rate of $17.5 \%$. Failure rate in their study was attributed to the lack of proper skill in the initial period of the study. In our study failure rate was due to patients not tolerating the manipulation in the growth of vocal cords and in the postcricoid region. However, examination in these patients was complete but they did not allow to take the biopsy. Procedure was abandoned due to poor patient comfortable score and unstable hemodynamic parameter. Breathing and voice usually remained normal. Patients complained about the heaviness or numbness in the throat as distressing to them. These patients are well counselled and reassured about this phenomenon being temporary due to the effect of topical anaesthesia. There could be loss of pitch while speaking loudly due to inadvertent anaesthesia of external branch of the superior laryngeal branch and impairement of deglutition ${ }^{[8]}$.

\section{Conclusion}

Direct Laryngoscopy under topical anesthesia and superior laryngeal nerve block becomes easy to perform and comfortable to the patients. Patients presenting with supraglottic and hypopharyngeal growths can be undertaken for direct laryngoscopy and biopsy using this technique. This technique is a good alternative to the general anesthesia and can avoid any undue delay in management and treatment of the patients. This technique of regional anesthesia for direct laryngoscopy has minimal haemodynamic instability in the patients, effective anesthesia and is associated with little risk to the patients.

\section{Conflict of interest}

None

\section{Funding Statement}

Source of funding- Nil

\section{Acknowledgement}

Nil

\section{References}

[1] Trevedi V, Sharma G. Evaluation Of Airway Blocks Versus General Anaesthesia For Diagnostic Direct Laryngoscopy And Biopsy For Carcinoma Larynx. J Indian Med Assoc. 2012; 110: 78-83.

[2] English J Norris A, Bedforth N. Anaesthesia For Airway Surgery. Continuing Edu Anaesthesia. Crit Care Pain 2006; 6(1): 28-31.

[3] Faccenda Ka, Finucane Bt. Complications of Regional Anaesthesia: Incidence and Prevention. Drug Saf. 2001; 24: 413-442.

[4] Auroy Y, Narchi P, Messiah A, Et Al: Serious Complications Related to Regional Anaesthesia: Results Of prospective Survey in France. Anaesthesiology 1997; 87: 372-383.

[5] Gotta A W, Sullivan C A: Anaesthesia Of Upper Airway Using Topical Anaesthetic And Superior Laryngeal Nerve Block. British Journal of Anaesthesia. 1981; 53: 1055.

[6] Chatrath V, Sharan R, Jain P, Bala A, Sudha R .The Efficacy Of Combined Regional Nerve Blocks In Awake Orotracheal Fiberoptic Intubation. Anesthesia: Essays and Researches. 2016; 10(2); 255-61.

[7] Shivkumar RK, Rajaseker R, Sellappan P, Anandan Heber. Efficacy of Bilateral Superior Laryngeal Nerve Block for Diagnostic Direct Laryngoscopy: International Journal of Scientific Study. 2017; 4(11): 132-135.

[8] Sulica L, Blitzer A. Anaesthesia for Laryngeal Surgery in the Office. The Laryngoscope. 2000; 110: 1777- 79.

[9] Bastian RW, Delsuphehe KG. Indirect larynx and pharynx surgery: a replacement for direct laryngoscopy. Laryngoscope. 1996; 106: 1280- 86.

[10] Hogikyan ND. Transnasal endoscopic examination of the subglottis and trachea using topical anaesthesia in the otolaryngology clinic. Laryngoscope. 1999; 109: 117073.

[11] Curran J, Hamilton C, Taylor T. Topical Analgesia before Tracheal Intubation. Anaesthesia .1975; 30: 7657. 